\title{
WURN TECHNIQUE IN GYNAECOLOGICAL NURSING CARE
}

\author{
M. Annie Annal, B.Anitha. *
}

\begin{abstract}
The Wurn technique is a manual physical therapy technique used as a form of alternative medicine gives often dramatic improvements in urogenital, reproductive, sexual function, and to treat endometriosis, pelvic inflammatory disease, pelvic spasms, polyps, and tubal obstruction. The wurn technique focuses on decreasing pain and increasing mobility and function of abdomino-pelvic and reproductive organs by diminishing adhesions.
\end{abstract}

Key Words : Adhesion, Endometriosis, Infertility, Pelvic inflammatory disease, Pelvic spasm, Technique.

\section{Introduction}

The Wurn technique is a site-specific massage therapy in the form of relaxing technique. It is designed to deform and detach the bond of adhesions and return the body to normal, painfree function of reproductive organs. ${ }^{1}$ This physical therapy focuses on deforming the adhesive collagen cross-links that comprise adhesions and appear to contribute to treat the underlying causes of infertility, including mechanical blockages and some hormonal imbalances ${ }^{2}$. By decreasing adhesions that bind the organs appears to help the body to function and

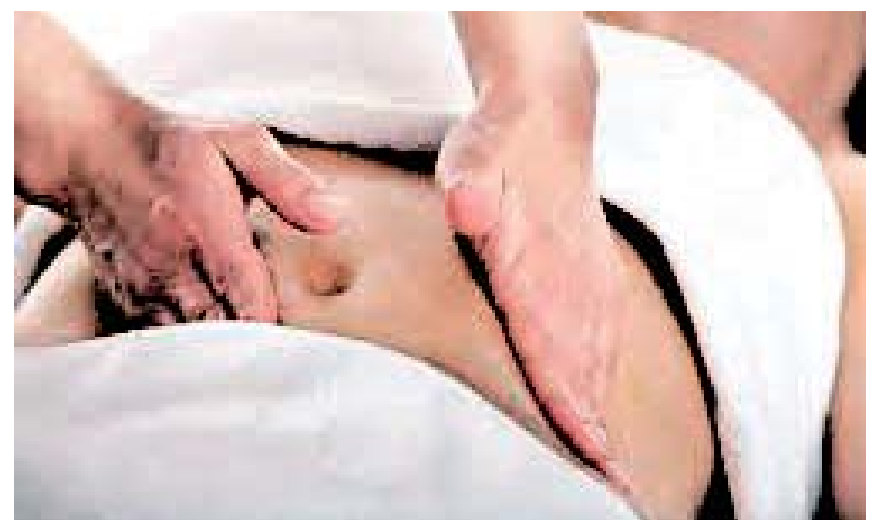

Wurn technique to promote the function of abdomino-pelvic and reproductive functions.

Wurn technique is a unique therapy, developed by physical therapist Belinda Wurn \& Larry Wurn. The nurses must require training courses from physical therapist and must be licensed to treat the gynaecological problems with wurn technique. ${ }^{3}$

\section{Purposes:}

The principle intent of the Wurn Technique is to find adhered tissues and structures wherever they exist in the body and detach the chemical bond of adhesions, thereby it helps,

Do improve fertility and improve pregnancy rates

$>$ To help in opening Blocked Fallopian Tubes

$>$ To increase Orgasm and treat inhibited Orgasm, dyspareunia and other sexual dysfunctions

$>$ To treat Endometriosis

$>$ To decrease pain and restore mobility after surgical procedure by improving soft tissue mobility, elasticity and distensibility

$>$ To improve circulation and restore balance, functional ability of reproductive organs

* Prof. M. Annie Annal, HOD, Ms .Anitha,B, Lecturer, Dept. of OBG Nursing, KGNC, Puducherry 607402, India. 
To correct Postural asymmetries or deviations To alleviate stress and induce overall relaxation ${ }^{1}$.

\section{Adhesion}

Adhesions are scar tissue resulting from infection, inflammation, trauma, or sugery occurring anywhere in the body. They are tiny but very strong collagen fibres that form naturally as the firststep in healing.It is the body's response to surgery and the healing process in the presence of increased levels of inflammatory cytokines that promotes the formation of adhesions ${ }^{4}$. They are bands of scar tissue with the potential to bind organs to other structures, which leads to multiple symptoms including organ dysfunction and pain. It reduces the function and movement of affected organs. In addition to being a common outcome of pelvic or abdominal surgery, adhesions are known to accompany related conditions such as bowel obstruction, chronic abdomino pelvic pain, endometriosis, pelvic inflammatory disease, pelvic spasms, polyps, and tubal obstruction. Dyspareunia and orgasmic problems may be due to the formation of pelvic adhesions.

Adhesions can restrict the mobility and function of the organs, ligaments, muscles and nerves of reproductive organs. Thus, they affect the biomechanics of the entire abdomino pelvic region, limiting the ability to conceive even with In vitro fertilization (IVF) and other assisted reproductive technologies (ART) ${ }^{5}$.

\section{Effect of Wurn Technique in Adhesion:}

The therapy used various site-specific pressures across the restrictive bands of adhered tissues and structures, working progressively deeper from the most superficial tissues, to restore mobility of structures. The wurn technique operates by peeling apart adhesions. The Nurse should apply site-specific pressure over the adhered part in the abdominal region over the courses of 20 hours in five days It improves the movement and elastic nature of the targeted reproductive organs and various support structures that hold them in place. The soft tissue pressure application decreases the amount of adhesions and micro adhesions in the uterus and uterine wall. Mobilization of the soft tissue may break down collagenous cross-links and adhesions that can cause pain and dysfunction including dyspareunia and inhibited or absence of orgasms. This reduction in adhesion makes it easier for an embryo to implant by improving the surface of the uterus. It reduces spasms in the uterus and cervical stenosis.

\section{Infertility}

Infertility is defined as the inability to conceive after 12 months of unprotected sexual intercourse. Internationally, the time frame is generally longer than 24 months. Approximately 5 million infertile women in the United States, it is estimated that 2 million (40\%) have medical or hormonal infertility; 1 million (20\%) have idiopathic infertility; and 2 million (40\%) have mechanical infertility.

Pelvic adhesions are the primary causes of mechanical infertility. In addition to being a common outcome of pelvic surgery, the formation of pelvic adhesions is known to accompany related conditions such as endometriosis, pelvic inflammatory disease (PID), tubal obstruction, polyps, pelvic spasms, bowel obstruction, and chronic abdominopelvic pain. ${ }^{[6-8]}$ Infertility-causing adhesions may form in the following locations,

- on uterine walls and ligaments, increasing the possibility of uterine spasm, implantation problems, and miscarriage and decreasing the ability to conceive;

- at and within the tissues of the cervix, creating stenosis, affecting the relaxed midline position, contributing to uterine spasms, and complicating sperm transfer to the uterus;

- on the surface of the ovaries, preventing exposure of the ovum and making transfer to the fallopian tube difficult;

- at the distal aspect of the fallopian tube, restricting the tentacle-like grasping of the egg by the fimbria, hence increasing its risk of being wasted in the abdominal cavity; and

- anywhere on the inside or outside of the fallopian tube, causing partial or total tubal occlusion, decreasing the probability of conception, and increasing the chance of an ectopic pregnancy. 


\section{Effect of Wurn Technique in Infertility:}

This manual therapy appears to be beneficial for women diagnosed with adhesions, potentially improving conception rates for women using this technique. Among women with the above-listed causes of infertility, the adhesions tend to block organs that are critical for the reproductive process. This includes the fallopian tubes and ovaries. In Wurn Technique Nurse practioner applied pressure to the specific identified site for the courses of 20 hours in five days programme. This approaches reduce the incidence of infertility through treating adhesions.

This technique uses as a natural infertility treatment, this therapy functions as an adjunct to regular gynecologic care when applied before intrauterine insemination (IUI) and IVF. It helps to improve mobility and motility of the reproductive organs by decreasing the following:

- adhesions and microadhesions on and within the uterine walls, helping to create a more hospitable surface for implantation;

- uterine and cervical hypertonicity and spasm, thus creating a more relaxed environment for implantation;

- cervical stenosis, adhesions, and tensions within the cervix and its attachments, thus improving cervical mobility and facilitating transfer to the preferred implantation site.

- Thus, In wurn technique, the adhesions are resolved, the infertility can also be cured and reproductive health can be restored.

\section{Endometriosis}

Endometriosis often accompanied by adhesions. Adhesions from endometriosis can join structures with strong glue-like bonds. Adhesions, endometriosis, pain and dysfunction are intimately related. Advvhesions from endometriosis can cause pain anywhere in the body (abdomen, pelvis, lower back). Dysfunctions such as poor digestion, irritable bowel and infertility may also result from the adhesions and endometriosis.

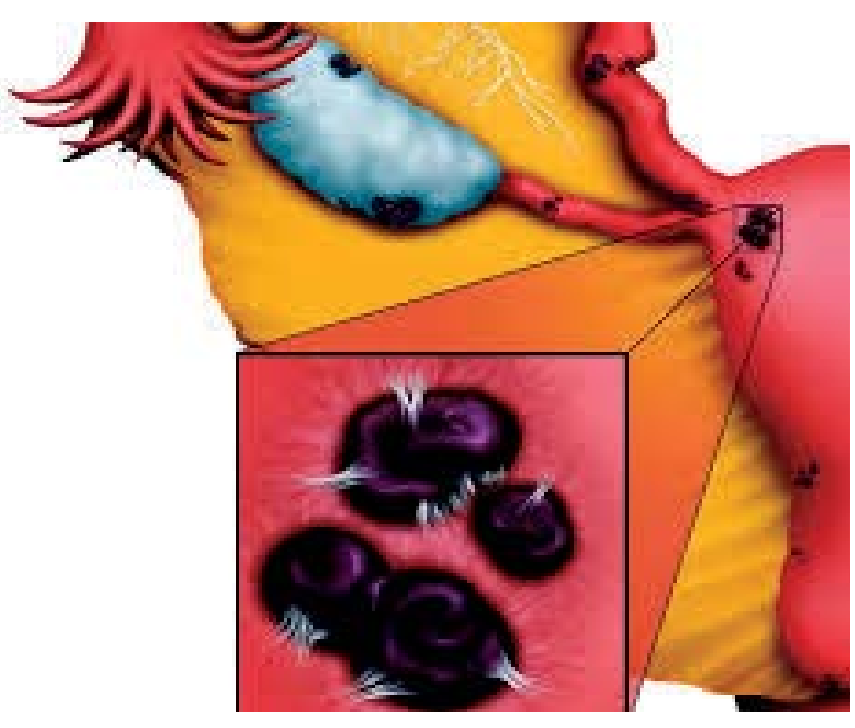

Endometriosis with Adhesions

Adhesion may form as a result of endometrial implants bleeding into the area which cause inflammation, leads to to the formation of scar tissue as a part of the healing process. Patients may develop adhesions after laproscopic excision of endometriosis. The glue-like bonding of adhesions has potential to cause decreased mobility and motility of abdominal, pelvic organs, pain, and dysfunction of adhered structures.

\section{Effect of Wurn Technique in Endometriosis}

The Site-specific manual Wurn Technique can improve soft tissue elasticity, mobility and distensibility and rendering improvement in reproductive rates. This massaging technique resolves the adhesion which around the structures and associated symptoms of endometriosis ${ }^{7}$.

\section{Blocked Fallopian Tube}

Blocked Fallopian tube is a major cause for female infertility. Blocked fallopian tubes are unable to let the ovum and the sperm coverage, thus making fertilization impossible. 


\section{Effect of Wurn Technique in Blocked Fallopian Tube}

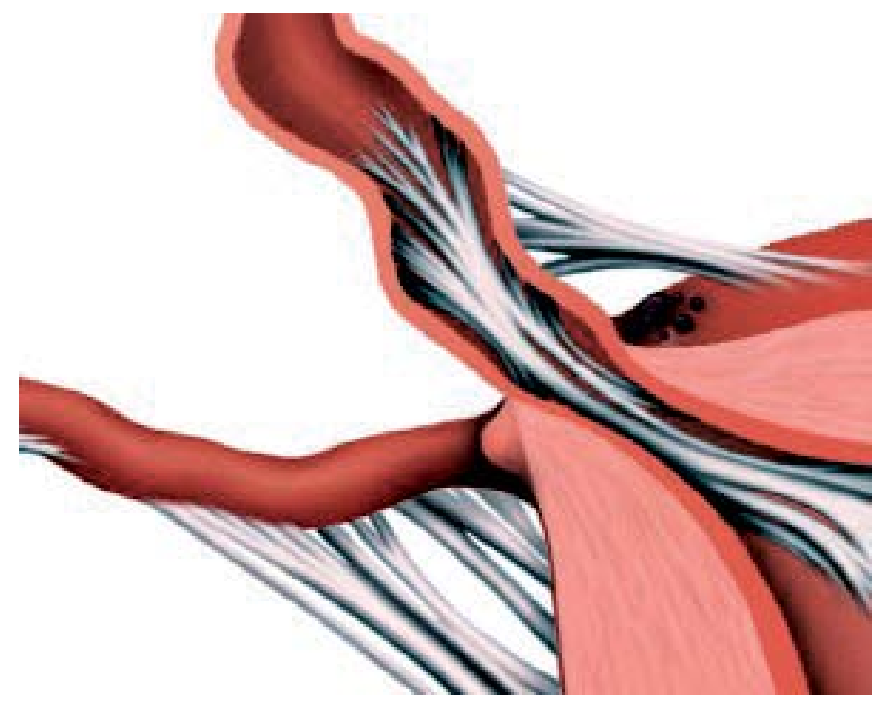

When adhesions block the tube by the uterus, nothing can pass. Eg .sperm, egg

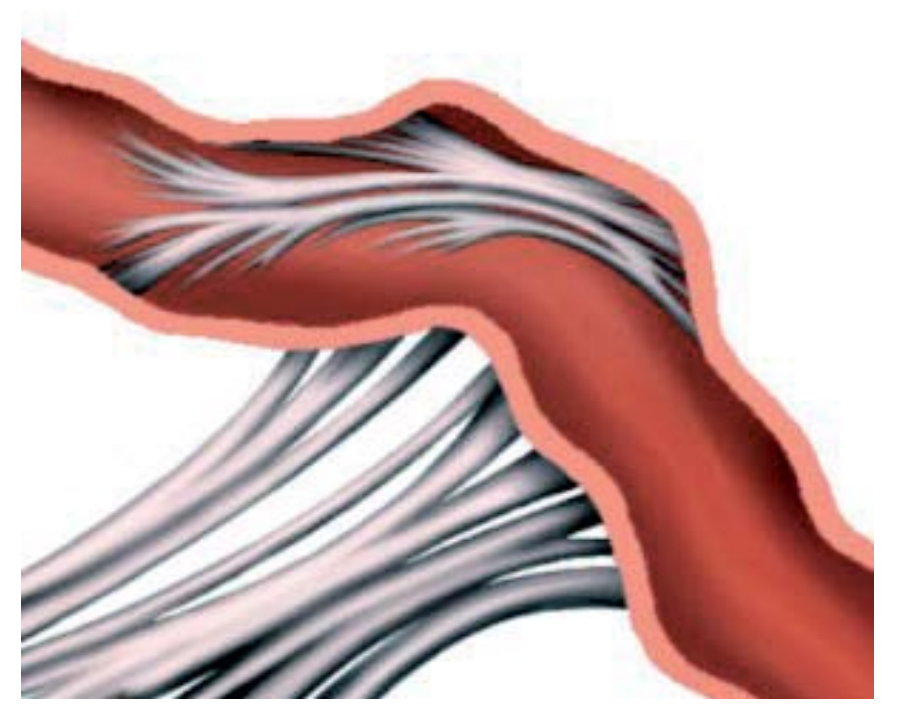

The therapy also cleared tubes blocked by adhesions in the tube and function returns to normal

\section{Nurses Role in Wurn Technique:}

- Extensive Patient History Review

- Motivate for Patient Participation

- Thorough Palpatory Evaluation

- Manual Treatment

\section{Extensive Patient History Review}

- Nurse should receive the patien t's historyincluding, gynecologic, surgical history

- Collect and note thorough past history of trauma, surgery, infection and inflammation.

\section{Motivate for Patient Participation}

- Nurse should motivate the client for therapy. Ensure that the wurn technique is a nonsurgical technique without adverse effect for the client.

\section{Thorough Palpatory Evaluation}

- Nurse must do the palpatory assessment of the patient's abdomen and pelvis, specific areas of visceral and myofascial cross-linking are determined the adhesion sites due to their restricted mobility ${ }^{9}$.

\section{Manual Treatment}

Focusing on these adhered areas, the therapist engages the uterine fundus and sidewalls and tractions them to the left. To assist and improve the mobility of the soft tissues, the therapist may release the tension of the traction either suddenly or gradually, depending on the desired effect to decrease spasm and adhesions between the uterus and the bladder.

This was evidenced by increased mobility at the precise sites of visceral and myofascial restrictions after each therapy session. The changes were further demonstrated by improved alignment, biomechanics, and increased range of motion of osseous and soft-tissue structures (eg, improved pelvic floor

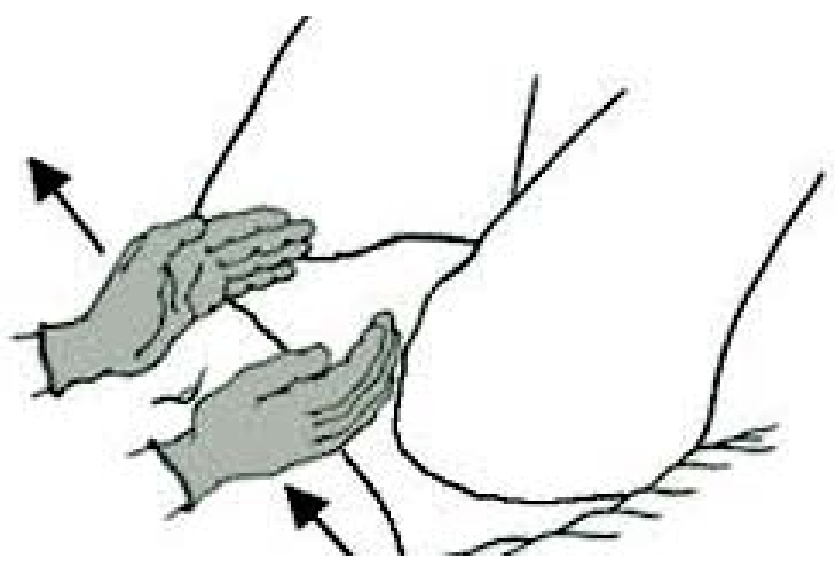


musculature tone, decreased pelvic floor spasms) (10-12).

This site-specific manual soft-tissue therapy improves soft-tissue mobility, elasticity, and distensibility. Mobilization of the soft tissue may break collagenous cross-links and adhesions that cause pain and dysfunction, including physiciandiagnosed mechanical infertility ${ }^{14}$.

\section{Conclusion}

The primary goals of manual therapy are to decrease pain and restore mobility by improving soft-tissue mobility, elasticity, and distensibility. The intent of the manual physical therapy protocol used to create microfailure of collagenous cross-links, the "building blocks" of adhesions ${ }^{13}$. These unique softtissue techniques were developed after extensive study of current, innovative, manual physical therapy methods.

The Wurn method of massage helps in softening and stretching the adhesions. The idea is to address any kind of adhesion-related problem, including overstretched, adhered or restricted connective tissue. The massage attempts to restore proper circulation among the affected areas. The Wurn philosophy believes that the adhesions are composed of tensile, collagen fibers that form layers of scar tissue. Thus, this therapy treats adhesion related conditions and promotes reproductive health and increase conception rates through massaging ${ }^{15-16}$.

\section{References}

1. Wurn BF, Wurn LJ, King CR, Heuer MA, Roscow AS, Scharf ES, Shuster JJ. Treating Female Infertility and Improving IVF Pregnancy Rates with a Manual Physical Therapy Technique. Med. Gen. Med. 2004 Jun 18; 6(2): 51. PMID 15266276

2. Burnette, A. Physical Therapy to Improve IVF Pregnancy
Rates. Achieving Families. 2005, Sept: 30.

3. Liakakos T, Thomakos N, Fine PM, Dervenis C, Young RL. Peritoneal Adhesions: Etiology, Pathophysiology, and Clinical Significance. Dig Surg. 2001; 18: 260-273. PMID 11528133.

4. Ellis H, Moran BJ, Thompson JN, Parker MC. Adhesionrelated hospital readmissions after abdominal and pelvic surgery: a retrospective cohort study. Lancet Br J Med. 1999; 353: 1476-80. PMID 10232313

5. Mosher W, Pratt W. Fecundity and Infertility in the United States. 1990.(Dec 4) 2-6. Available from: http:// www.clearpassage.com/what-we-treat/infertility/blockedfallopian-tubes/

6. Meldrum DR, Hacker NF, Moore JG. Essentials of Obstetrics and Gynaecology.Philadelphia.W.B.Saunde rs.1992:444.

7. Stickler RC.Factors influencing fertility-Evaluation and Treatment. Philadelphia. W.B saunders.1995:8-18.http:// www.livingwithendometriosis.org/category/alternativemedicine/

8. Stone K. Adhesions in Gynaecologic surgery. Curr opin obstet Gynaecolo. 1993:5:322 Available from: https:// diaryofamiracle.wordpress.com/2009/06/21/what-is-thewurn-technique/

9. Stege JF, Stout Al. Resolution of chronic pelvic pain after laproscopic lysis of adhesions. AM J crynecol.1991: 165: 278-283. Available from: https://www.linkedin.com/pub/ belinda-wurn/9/a41/9a1

10. Pt Belinda Wurn,Lmt Larry Wurn, MD Richard King . 2009 - Health \& Fitness

11. Valiani, M. The effects of massage therapy on dysmenorrheal caused by endometriosis. Iran J Nurs Midwifery Res: 2010;167-71. Available from: http://www.clearpassage.com/ who-we-are/about-the-wurn-technique/.

12. Juliafracegiaslmt. 2015.(April) Available from: https:// diaryofamiracle.wordpress.com/2009/.../what-is-the-wurntechnique...

13. Lawrence J Wurn, LMT, Belinda F Wurn, PT, and Amanda $\mathrm{S}$ Roscow, MPT. Increasing Orgasm and Decreasing Dyspareunia by a Manual Physical Therapy Technique: Available from: http://www.wisegeek.com/what-is-thewurn-technique.htm\#didyouknowout.

14. Holtz G. Prevention and management of peritoneal adhesions. 1984: 41: 497-507.

15. Wiechmann G. Wurn L. Manual soft tissue therapy to decrease Abdomino pelvic adhesions.Gianservicle. http:// infertilityhandbook.com.au/?p=568

16. Drollette CM, Badawy SZ. Pathophysiology of pelvic adhesions.Modern trends in preventing infertility. J Reprod med. 1992. 37: 107-122. 